\title{
ENFERMEDAD MENTAL Y MARGINACIÓN. APROXIMACIÓN HISTÓRICA A LA INCAPACIDAD CIUDADANA EN CHILE, SIGLO XIX.
}

\author{
MENTAL ILLNESS AND MARGINALIZATION \\ A HISTORICAL APPROACH TO CIVIC INCAPACITY IN CHILE, 19TH CENTURY.
}

\section{María José Correa Gómez ${ }^{1}$}

\begin{abstract}
Resumen:
Este estudio se pregunta por la relación entre enfermedad mental e incapacidad en el Chile republicano del siglo XIX. Para ello revisa las formas de administración de la locura, centrándose en la interdicción por demencia. Mediante la revisión en profundidad de expedientes judiciales de interdicción guardados en el Archivo Nacional Histórico, introduce a los contextos cotidianos que fueron ubicando a la locura como enfermedad y a esta como sustrato de incapacidad, en décadas de organización ciudadana y de institucionalización del conocimiento científico. El estudio identifica los pretextos y contextos que fueron marginando a ciudadanos, aparentemente problemáticos e improductivos, y revela que las dificultades para adscribirse a las condiciones de la vida urbana y acatar los principios económicos de la modernidad, representaron importantes conectores de las nociones de enfermedad e incapacidad.
\end{abstract}

\section{Palabras claves:}

enfermedades mentales, incapacidad, interdicción, Chile, siglo XIX

\begin{abstract}
:
This study explores the relationship between mental illness and incapacity in 19th century Chile. It examines the management of insanity by focusing on mental interdiction. Through the analysis of interdiction legal records kept at the Archivo Nacional Histórico, the paper introduces both the daily forces that influence the transformation of madness in an illness, and the use of disease as an explanatory frame of incapacity, in a period portrayed by the organization of citizenship and the institutionalization of scientific knowledge. The study identifies the excuses and the contexts that promoted the social exclusion of apparently difficult and unproductive citizens. Theses traces how that the problems to follow the challenges of urban life and to accept the economic principles of modernity, represented pivotal connect or sof the notions of disease and incapacity.
\end{abstract}

\section{Keywords:}

mental disorders, incapacity, interdiction, Chile, 19th century

Historiadora, Doctora en Historia UCL, Académica Universidad Andrés Bello, Licenciatura en Historia e Investigadora Postdoctoral Conicyt, proyecto 3130335. Campus Viña del Mar, Quillota 910. maria.correa@unab.cl. 
Durante el siglo XIX la locura modificó su estatus y su posición en la sociedad chilena. La institucionalización del saber médico, tras la creación de la Escuela de Medicina en 1833,amplió sus condiciones de existencia, mientras que el conocimiento de la enfermedad mental por medio de la disciplina alienista, y luego psiquiátrica, renovóla confianza en la redención terapéutica y, como resultado, cuestionó el oscuro destino que presagiaba la pérdida de la razón.

La defensa del carácter patológico de la locura por parte de la ciencia médica, estuvo acompañada del reconocimiento de la razón como uno de los bienes más preciados del proyecto ilustrado decimonónico. Durante este periodo la razón emergió como base del programa ciudadano y como requisito del adelanto y del progreso. En este afán, la locura cobró visibilidad como posibilidad desestabilizadora del precario orden nacional, como metáfora del descontrol, como alegoría del caos y como afrenta al orden social.

El riesgo asociado a la locura impulsó su tutela. La razón fue medida y estudiada, vigilada y judicializada con el objeto de identificar sus quiebres y administrar sus excesos. La razón enferma fue abordada médicamente a través de los sistemas curativos introducidos por el alienismo-simbolizadosen la Casa de Orates y en las nuevas terapéuticas- y también, por medio de los marcos legales que ordenaron el lugar de la mente enferma en la sociedad chilena. Bajo estas premisas, un importante número de hombres y mujeres enfrentó cuestionamientos a su sanidad mental, como resultado de comportamientos y fisonomías que parecían alejarse de una normalidad en proceso de definición. Histerias, demencias seniles, parálisis, arterioesclerosis, alcoholismos, fatuidades, idiotismos, congestiones, alucinaciones, delirios, sífilis, epilepsias y paranoias, entre otros diagnósticos, contribuyeron a respaldar estas imputaciones y operaron como engranajes conceptuales de la instalación del ideario alienista o pre-psiquiátrico en Chile.
Sin embargo la locura no solo trajo consigo la imputación de un diagnóstico y la clasificación de ciertas personas como insanas. También acarreó consecuencias concretas sobre los enfermos y sus entornos, que se manifestaron en desplazamientos y nuevos estados. La constatación de una enfermedad mental facilitó la adscripción del insano al tratamiento moral, y también permitió la restricción de los derechos ciudadanos por medio de la interdicción.

Este artículo examina la construcción histórica de la incapacidad desde las formas de administración de la locura y de sus problemas, por parte de la sociedad chilena del siglo XIX. Considera la estructura legal republicana que -apoyada en legislaciones internacionales decimonónicas y en las Siete Partidas (Siglos XIII-XIV)-definió a los dementes como incapaces y sugirió ubicarlos bajo la curatela de un tercero. Al mismo tiempo, introduce a aquellos cambios en el saber y en la institucionalidad médica que,ayudando a posicionar y difundir una mirada científica en desmedro de explicaciones demonológicas, respaldaron la falta de capacidad de los dementes. También aborda transformaciones sociales más amplias,inscritas en procesos de capitalización quer eforzaron la marginación de los enfermos mental es en la administración de sus asuntos patrimoniales y económicos.

Así, el presente estudio examina la noción de incapacidad mental por medio de la interdicción por demencia, atendiendo a los modos, como ha planteado Eugenia Molina, en que una comunidad, en un determinado momento, se relacionó con la ciudadanía, "lo que hace o permite hacer con ella una sociedad»(Molina, 2002, 136). El análisis se detiene particularmente en el estatus de incapacidad asociado a la demencia legal,con el objeto de problematizar las relaciones entre enfermedad mental y modernidad. Para ello, hace uso de un variado grupo de registros primarios que van desde estudios de abogados y médicos, a los expedientes de curatela de dementes y de 
interdicción de los archivos judiciales del Archivo Nacional Histórico de Chile².

\section{La República y la capacidad de sus ciudadanos}

El interés por determinar la capacidad de las personas y administrar sus acciones marcó la temprana organización de la República. La Constitución de 1833 ofreció igualdad ante la ley, libertad de permanecer y trasladarse por el país, derecho de propiedad, derecho de presentar peticiones a las autoridades y libertad de expresión;sin embargo, en la práctica, estos beneficios estuvieron reservados para todos quienes cumpliesen los requisitos de elegibilidad. Así, la búsqueda por la igualdad y la libertad derivó en la imposibilidad de muchos de acceder a los beneficios ciudadanos prometidos (Cansanello, 2003). Ejemplo de estas distancias fueron las condicionesrequeridas para el ejercicio de la ciudadanía activa, que consideraba el derecho a sufragio. Esta estaba reservada solo a los chilenos mayores de veinticinco años si eran solteros o veintiuno si se encontraban casados, que además de saber leer y escribir, contaran con una propiedad inmueble o un capital invertido, o en caso contrario, que ejercieran alguna industria o arte o tuviesen un empleo. Estas exigencias llevaron a la restricción de la ciudadanía activa, alcanzadaen 1846 por soloun $2 \%$ de la población (Collier, 2003).

Este estudio corresponde a una prolongación de la investigación doctoral realizada entre los años 2007 y 2012 en Wellcome Trust Centre for the History of Medicina, University College London y contó con el apoyo de WT Studentship y UCL Overseas Research Students Award. Dicha investigación abordó la medicalización de la locura desde el estudio en profundidad de alrededor de 350 expedientes de curatela e interdicción por demencia de distintos juzgados de Chile entre 1830 y 1920. Pese a que estos corresponden a todos los pleitos identificados en el Archivo Histórico Nacional, según los catálogos disponibles para ese periodo, no reflejan la totalidad de la práctica judicial de curatela e interdicción. Otros registros, como las inscripciones del Conservador de Bienes Raíces, dan cuenta que por ejemplo, para la segunda mitad del siglo XIX existieron al menos 1.000 inscripciones de interdicción en Chile.
Al pequeño alcance de esta prerrogativa, se sumaron las formas de exclusión basadas en la capacidad de las personas. El Código estableció que la calidad de ciudadano activo se suspendía por haIlarse procesado por delito, por tener deudas con el fisco, por la condición de sirviente doméstico y por "ineptitud física o moral" que impidiese" obrar libre y reflexivamente" (Constitución, 1833, 4). Esta última condición mostró los significados inscritos en lo patológico y su vinculación con la ciudadanía, entendida esta última, como el modo de relación entre los individuos y el Estado sobre la base de un imaginario de coparticipación (Sábato, 1999).Al plantear que la incapacidad física o moral llevaba a la supresión de la cooperación, el Estado significó una serie de difusos fenómenos corporales y mentales y caracterizó a quienes los experimentaran como personas problemáticas que demandaban un trato particular.

El ideario republicano mostró un discurso contradictorio que en ocasiones aplaudió la igualdad y la libertad individual, y en otras reforzó la segregación. Esta tensión se materializó en el texto constitucional de 1833 y posteriormente en los cuerpos legales que establecieron condiciones diferenciadas para sus ciudadanos, relegando a un vasto número de hombres y mujeres en razón de su cuerpo y de su mente. Posteriormente, el Código Civil, en aplicación desde 1857, reconoció la importancia de ordenar aspectos privados en posde la conservación del orden y de la estabilidad nacional. Consideró que las diferencias físicas y mentales impedían la correcta realización de los actos jurídicos y definió a los sujetos que tuviesen estos atributos como "dependientes» e «incapaces». Estableció que los sordos-mudos que no podían darse a entender por escrito, los impúberes y los dementes debían estar bajo interdicción y prescindir de su habilidad para administrarse a si mismos, legitimando la marginación de «la ineptitud» ciudadana proyectada por la Constitución de 1833. Estas normas tensionaron la supuesta capacidad adscrita al cuerpo y a la mente de los ciudadanos, al mismo tiempo que confirmaron ciertos comportamientos y estados como con- 
diciones transgresoras de la ciudadanía (Correa, 2013, 18-19).

A diferencia de la Constitución de 1833, que abordó el problema de la locura indirectamente, asociándolo a desarreglosmorales y a un mal usode las facultades individuales, el Código Civil se refirió expresamente a la demencia.Pese a que codificaciones anteriores, como las Siete Partidas, habían regulado los derechos de los «lunáticos», el texto de Bello subrayó la importancia de diferenciar entre el incapaz y el indigno (Bello, 1874). Para ello hizo uso de la interdicción, una herramienta legal más poderosa y persistente que el encierro terapéutico -este último frágil e intermitente durante la segunda mitad del siglo XIX, quepermitía cambiar el estado de un individuo diagnosticado en un estado habitual de «demencia», declarándolo incapaz de los actos de la vida civil, privándole de la administración de sus bienes y de la libertad de disponer de su persona (De la Plata, 1887)3. La declaración de una persona como interdicta teníaimplicancias profundas. Entre las muchas consecuencias de la oficialización de una demencia por medio de la interdicción, estaban la restricción de los derechos individuales y la pérdida de la capacidad jurídica, lo que generaba la neutralización de la personalidad legal del demente y el traspaso de la administración de su persona y bienes a un curador.

La noción de demencia se empleaba en la justicia "por analogía para significar todos los casos en que la razón no puede manifestarse con perfecta conciencia de las acciones" ${ }^{\prime 4}$. No hacía alusión a las nuevas nosologías que gradualmente entraban

Como planteaba de la Plata, el términodemencia en el ámbito legal se usaba "poranalogíaparasignificartodos los casos en que la razón no puedemanifestarse con perfecta conciencia de lasacciones".

4 Por el contrario, la palabra demencia desde el ideario médico tendía a explicar el debilitamiento de las facultades intelectuales a causa de la edad avanzada, sin embargo en el derecho se empleaba este término "por analogía para significar todos los casos en que la razón no puede manifestarse con perfecta conciencia de las acciones" (De la Plata, 1887, 5) al ámbito médico, sino a un estado habitual de enfermedad mental que respondía sin problemas a la cambiante y móvil red de enfermedades acuñadas con el desarrollo del alienismo. Esta falta de definición en torno a los estados de demencia y su relación con la capacidad, ofreció a los usuarios de la justicia una mayor flexibilidad al momento de utilizar la interdicción, descartando la exigencia de un lenguaje preciso y permitiendo demandarun estado de incapacidad, sobre la base de conflictos, comportamientos y patologías que en ocasiones poco parecían tener que ver con la locura (Correa, 2013).

El uso de esta herramienta legal se vinculó con las nuevas necesidades que mediaban la relación entre los ciudadanos y el Estado.Con el tiempo, el desarrollo urbano, la mercantilización de la sociedad y la profesionalización del saber, renovaríanlos contextosde interpretación de la ciudadanía y, como consecuencia, promoverían demandas en el ejercicio de los derechos y deberes ciudadanos. Así, la capacidad enfrentó nuevos desafíos. La idea del ciudadano apto y capaz se relacionó estrechamente con la administración del capital, con la habilidad para participar en las estructuras de la vida urbana, con la idoneidad para continuar reproduciendo el capital económico de una familia, y con la disposicióna vivir en armonía en una comunidad densa y necesitada.

\section{La medicina, los médicos y el diagnóstico de la demencia}

El interés por la incapacidadse relacionó con el reconocimiento de distintos tipos de "imperfecciones" físicas e intelectuales en la población del país y con los procesos de cambio que introducía la modernidad.Esta alianza Ilevó a que los miembros de la comunidad médica nacional advirtieran, durante el curso del siglo, sobre los peligros del brote malsano que acompañaba el desarrollo de las grandes ciudades. La civilización moderna llegaba cargada de riesgos y desafíos que implicaban, en ocasiones, el despertar de trazos morbo- 
sos sobre los cuerpos. Como explicaba el organizador de la Escuela de Medicina, WilliamBlest, el desarrollo urbano había llevado a que los cuerpos y mentes ciudadanas se cubrieran de hastío, los rostros se volvieran "pálidos y túrbidos" tes se elevaranabatidas y los ojosmirasen lánguidamente (Blest, 1828, 9).

Esta debilidad que parecía afectar a la población chilena conforme avanzaba la civilización, fue tema recurrente en los escritos médicos de mediados del XIX. La comunidad científicaleyó la heterogeneidad social desde las fisonomías enfermas que resultaban de la imperfecta combinación de lugares, alimentos y costumbres y desdela fisiología frágil que obstaculizaba el crecimiento de las cualidades criollas. Como explicaba el médico José Juan Brunner,

"Chile mismo ve con dolor que su raza antes tan vigorosa se está debilitando gradualmente, que sus hijos mueren antes de llegar a la edad de la circunspección i consejo, que su población trabajadora se pone raquítica, que sus hijas envejecen antes del tiempo, i que la longevidad se encuentra tan solo en aquellos pocos que recuerdan las costumbres frugales de los padres de la patria" (Bruner, 1857, 308).

Esta mirada seguiría presente. El médico Wenceslao Díaz, algunos años después, insistiría en el retroceso que enfrentaba la constitución del pueblo chileno,

"ha cambiado tendiendo a hacerse de año en año mas débil, mas valetudinaria, mas raquítica. El trabajo ha crecido permaneciendo idénticos los alimentos, las habitaciones, los perversos hábitos hijénicos: el trabajo ha aumentado i los exesos de la embriaguez han aumentado tambien hasta tal punto que puede decirse que nuestra clase obrera

Se respeta la ortografía de lasfuentesprimarias. gana no para alimentarse, para proporcionarse todos aquellos medios que reparan o retardan el desgaste de la vida, sino para debilitar i destruir los resortes del organismo que la sostiene" (Díaz, 1863, 453).

Las autoridades compartían la mirada experta de los médicos y admitían que, como "en todas las poblaciones", existía en Chile una multitud de individuos que se hallaba "bajo condiciones vitales" que los hacían "inútiles (...) para la mayor parte de los trabajos u ocupaciones de la vida" (Memoria, 1859, 43).Por un lado se encontraban los que no podían trabajar producto de su edad, como niños y ancianos, y por otro, aquellos que tenían una enfermedad física o intelectual (Tagle, 1892-1893). En alianza con la beneficencia, el Estado ofrecía a los niños y a los viejos espacios orientados específicamente a ellos. Los primeros encontraron un lugar en la Casa de Maternidad anexada al Hospital San Borja o en la Casa de Expósitos, entregada a las Hermanas de la Providencia. Los segundos se paseaban por los pasillos de los precarios hospicios que se levantaban en el país y en otras casas de socorro. Junto a ellos sordos, ciegos, paralíticos, tullidos y locos, contribuían a ampliar el registro de los "imposibilitados", recordando el rol que debía cumplir la medicina y el Estado en su identificación y administración.

Como mostraban los resultados del censo hacia 1850, se identificaba "un individuo imposibilitado corporalmente por cada 89 habitantes, y un imposibilitado intelectualmente (loco o fatuo) por cada 772 habitantes". Esta cifra parecía exceder, según los entendidos, la de otros parajes, como el francés, donde existía "un imposibilitado físicamente por cada 137 habitantes y uno intelectualmente por cada 800 habitantes". La distancia generaba preguntas "¿Acaso entre nosotros los órganos de los sentidos o la razón individual son mas débiles e imperfectos?, ¿El clima, el método de vida i la constitución física pueden tener alguna influencia en la repetición de estos hechos?"(Memoria, 1859,43).A estas inquietudes se sumarían nuevas 
interrogantes que mostrarían las preocupaciones deuna nación que veía en su "población" la base de la "industria, la riqueza, el bienestar público, el poder, la gloria" (Anuario, 1868, vii).

Los médicos fueron reconocidos como especialistas para "estudiar estas cuestiones de tan vital importancia para la sociedad" y como los principales aliados del Estado en la identificación de aquellos trazos morbosos que delataban a los incapaces (Memoria, 1859, 43). No solofueron ellos quienes comunicaronel cuerpo teórico para comprender los fenómenos que desordenaban la moral y las facultades de los individuos, sino tambiénarticularon sus instrumentos con los estatales.En el ámbito de la locura, la especialización alienistay luego psiquiátrica, junto al desarrollo de espacios hospitalarios como la Casa de Orates de Santiago y la formulación de extensas nosologías médicascontribuyeron a reducir antiguas asociaciones de la locura yfrenar la reducción de los dementes a endemoniados o fatuos. Estos contextos permitieron profundizar el saber en torno a la enfermedad mental y también a vincular la ambición terapéutica con el afán de gobierno de la incapacidad.

Los médicos avalaron el desarrollo de una terapéutica específica para el control de la demencia, el tratamiento moral, planteado en Francia por Philippe Pinel (1745-1826) y posteriormente por Jean-Etienne Esquirol (1772-1840). Este modelo buscaba,como planteaba José Ramón Elguero (1819-1877), uno de los médicos a cargo de la institución, un aislamiento que separara al enfermo de su familia y que le permitiese dejar "aquellas relaciones i hábitos en medio de los cuales vive" (Elguero, 1863, 183) Posteriormente este espacio asilar permitiría implementar una paleta terapéutica más amplia, caracterizada por la aplicación hacia fines del XIX de la hidroterapia y de la electroterapia, y para inicios del XX de algunas propuestas de laborterapia, entre otros recursos.

Junto con confiar en esta vigilancia institucional de la demencia, los médicos dieron a conocer causas, progresos y prognosis de un creciente número de enfermedades mentales. La medicina relacionó la enfermedad mental con el temperamento y la constitución individual, con la herencia y la biología cerebral, así también con la fatiga y el estrés. Las experiencias de vida, los apremios, pesares, dolores; los golpes, carencias y excesos; el género, la edad y la clase, nutrieron el despertar y devenir de las condiciones mórbidas. En esta construcción de saber los especialistas ampliaron el sentido de la enfermedady la vincularon formalmente a las contextos cotidianos de hombres y mujeres.

El reconocimiento de los peligros que enfermaban a la población y frenabanel anhelado progreso, impulsó el usode instrumentosde identificación que complementaron, en aspectos sanitarios, otras acciones de empadronamiento.La comunidad médica mostró su propio interés en registrar y publicar los resultados estadísticos para "formar una idea de las enfermedades más comunes i de los tratamientos empleados en ellas con mejor éxito", mientras las autoridades vieron en los médicos a los expertos capaces de confirmar el grado de incapacidad de un ciudadano (Díaz, 1863, 455). Así, los médicos se transformaron en intérpretes de la relación que se establecía entre enfermedad y capacidad. Fueronvalorados por su conocimiento específico y ubicados como agentes obligados en la identificación judicial de una demencia, en un contexto que buscaba asegurar testimonios confiables y veraces por medio de la participación de personas que manejaran un determinado arte.

El Código Civil obligó que al menos dos médicos participaran en la identificación de la demencia en procesos deinterdicción. Esta confianza en la palabra especializada del facultativose cimentaba, por ejemplo en la ley de 1842,que impidió el ejercicio de la profesión científica a quienes no contasen con el grado de licenciado conferido o validado por la Universidad de Chile.

La participación médica en los juicios de interdicción pareció influir en la forma de nombrarla demencia. Vemos que previo a este requerimiento, cuando la identificación de la demenciasegún las normas compiladas en las Siete Partidas 
podía dictaminarse solo con la participación de testigos, los dementes fueron calificados por medio decondiciones generales y absolutas: fatuidad, enajenamiento, idiotismo, delirio y trastorno mental. La ausencia de facultativos sumado a un estado muy inaugural del saber médico académico en Chile, incidió en las descripciones y significaciones judiciales de la demencia. Posterior a 1857, la obligatoriedad de la información pericial en procesos de interdicción, incorporó a tribunales nuevas miradas que ampliaron las patologías descritas a melancolías, monomanías, histerias, epilepsias, delirios de persecución, alcoholismos, afecciones cerebro espinales, parálisis progresiva, entre varias otras (Correa, 2012). Pese a que este espectro patológico no desvinculó a la demencia con la incapacidad, si ofreció un renovado entendimiento de la enfermedad mental donde esta se explicaba no solo desde los problemas intelectuales, sino también desde las crisis emocionales y las fragilidades de la voluntad.

La medición de los ciudadanos y la ordenación de sus derechos y deberes de acuerdo al estado de su razón fueron instanciasavaladas por la ciencia médica y la práctica judicial. Como los propios legistas requerían, "la democracia moderna" no debía de acreditar otra incapacidad que aquella que venía de la edad o derivadas de problemas en las "facultades psíquicas a causa de trastornos mentales" y esta incapacidad debía establecerse "únicamente según los resultados de las ciencias que tienen por objeto el estudio de aquellas facultades" (Álvarez, 1901, 408). El problema radicaba en que la significación de la patología estaba teñida, en sus distintos momentos de formulación, por la equivalencia entre enfermedad mental e incapacidad.

\section{Enfermedad e incapacidad: sustantivos de la vida moderna}

La locura y la incapacidad no se explicaron tan solo desde la palabramédica. Las más de mil personas que acudieron durante la segunda mitad del siglo XIX a losjuzgados del país, como demandantes o testigos en casos de interdicción por demencia, dejaron en los expedientes narraciones sobre los contextos cotidianos de producción y significación de las enfermedades mentales.

Los demandantes se acercaron, en su mayoría, a solicitar laformalización de la incapacidad de parientes. Este fue el caso de Miguel Esquivel, quién acudió a los tribunales de Iquique hacia 1899 para pedir la interdicción de su padre, Marcelo Esquivel, por encontrarse "desde hace más de un año a esta parte" en estado de demencia e incapaz de administrar sus intereses. Habiéndose desempeñado como barretero en las minas y luego como jornalero, a sus ochenta años tenía "completamente perdida su cabeza, no pudiendo tampoco hacer uso de su cuerpo" y poca capacidad le quedaba, en palabras de su hijo, para "ocuparse en cualquier clase de trabajo o negocios por sencillos que sean" ${ }^{\prime 6}$. Los testigos confirmaron que no podía ocuparse en "trabajo alguno", que se perdía por las calles "casi siempre que sale solo", que se encuentra "casi ordinariamente sin conocimiento", "que está malo de la cabeza" y que "hai que andar vigilándolo". Sin embargo los médicos llamados por la justicia a evaluar el estado del imputado tuvieron una visión distinta de su capacidad. Los doctores del Río y Vargas Clark informaron al juez que pese a que sus facultades intelectuales se encontraban debilitadas y que su memoria necesitaba de un "grande esfuerzo para poder funcionar regularmente", contestaba "razonablemente" a lo que se le preguntaba. Añadieron que no se encontraba demente y que tenía el "discernimiento necesario" para saber quien podía ayudarlo a administrar convenientemente sus bienes ${ }^{7}$.

Miradas divergentes como las encontradas en el juicio de interdicción contra Marcelo Esquivel

Archivo Judicial de Iquique, (AJI) Declarandointerdicto al padre, 1899, L. 316, p. 17, f. 1 y 2.

AJI, Declarandointerdicto al padre, 1899, L. 316, p. 17, f. 9. 
abundan en el archivo y no solo entre legos y expertos, sino entre todos quienes se acercaron a intentar entender, clasificar y ordenar comportamientos y actitudes bajo el alero de la locura y la razón. La identificación de la locura no fue una tarea simple. Por un lado, las patologías ofrecidas por el alienismo a los médicos no eran fáciles de aplicar, requerían conocimiento, experiencia y tiempo. Significaba un enorme desafío el adscribir un determinado apellido a estados que no revelaban lesiones anatómicas aparentes, en un contexto donde las herramientas diagnósticas eran frágiles e inciertas y donde las tiempos judiciales invitaban a construir diagnósticos en tan solo un par de horas.

Esta divergencia trasluce además las distintas miradas que coexistieron en torno a la enfermedad y la incapacidad y permite trazar los distintos agentes de identificación e interpretación de la locura. Visibiliza el protagonismo de los legos, la actividad de quienes sin manejar un conocimiento experto de la ciencia médica, imputaron un estado patológico,abriendo el diálogo y la discusión. Pese a que ciertamente la disonancia representa un elemento transversal a muchas historias de interdicción, lo que resulta particularmente interesante es la constatación que la equivalencia entre enfermedad mental y falta de capacidad se gestaba en los hogares y en el vecindario, en el cotidiano que acompañaba a quienes parecían presentar un comportamiento "desviado".

Así, las alrededor de 350 demandas de interdicción revisadas para la segunda mitad del siglo XIX muestran que, pese al protagonismo del saber médico y de los propios facultativos en las investigaciones judiciales, la enfermedad mental se presentó primeramente desde lo doméstico y lo cotidiano y principalmente desde el incumplimiento de los deberes adscritos a los roles de género, etarios, de clase, laborales y domésticos. Como consecuencia,fue en el hogar y en la comunidad, donde las ideas sobre lacapacidad e incapacidad cobraron sentido asociadas a los desvíosidentificados enlos comportamientos de parientes o vecinos.
La demencia fue descrita ante la justicia desde las circunstancias cotidianasque demostraban la incapacidad de adecuarse a las responsabilidades de la vida urbana. Los demandantes y testigos hablaron de los enfermos como "incapaces", otorgándoles un género, una edad, un comportamiento y por sobre todo, una inhabilidad de responder a sus contratos y compromisos. Se refirieron a los "imposibilitados", "inútiles", "perturbados" y "perdidos". A través de las historias de vida y de las condiciones que acompañaban el levantamiento del juicio presentaron los comportamientos enajenados de los imputados y los modos en que la locura marchitaba la capacidad.De este modo, el "estado de demente" del joven italiano Cayetano Revellofue explicado hacia 1871 por su tío a través del abandono que este había hecho de un buque en la bahía de Valparaíso, por más de tres meses $^{8}$. La enfermedad de Miguel Carrasco fuemostrada por su esposa hacia 1877 en la complacencia de "acompañarse en la bebida con cualquiera persona por desconocida que sea, proporcionándola a su costa, habiendo sucedido no hace mucho, que compró un tonel de cuarenta arrobas de licor para que de el bebiera todo el que quería el resultado necesario fué pues que se concluyó en mui pocos días i sin que hubiera aprovechado sus arrobas siquiera" ${ }^{\prime 9}$.Así también, la locura de Delfina Araya fue retratada por su marido,en 1881, desde el viaje realizado a Santiago"con un niño de un mes llevándose a la criatura sin que siquiera se la pusiera bautismo"10.

La locura fue narrada desde los nuevosrequerimientos de la vida citadina de fines del siglo XIX. La acelerada urbanización modificó las condiciones materiales y las prácticas sociales.La población se elevó, particularmente la urbana. Los ran-

8 Archivo Judicial de Valparaíso (AJV),Interdicción, 1871, L. 1169, p. 7

9 Archivo Judicial de Rancagua, Sobreque se le declare en interdicciónparaadministrarsusbienes, 1877, L. 380 , p. 15 , f. $1 \mathrm{v}$.

10 AJV, Interdicción, 1881, L. 1168, p. 13, f. 1. 
chos rodearon las ciudades, mientras que cientos de conventillos, compuestos por una sucesión de habitaciones, se elevaron en los centros y acogieron, para el caso de Santiago, a alrededor del $40 \%$ de la población (Rengifo, 2012). La densidad poblacional vino acompañada por el despertar de nuevas tecnologías y ciencias que modificaron el rostro de Chile. Desde 1863 el ferrocarril comenzó a unir el país, cruzando pueblos y ciudades. Décadas más tardes, a inicios del XX, los primeros tranvías eléctricos se desplazarían por la capital, colaborando en la modificación de los tiempos y de las velocidades urbanas (De Ramón, 2000).

La historiografía ha dado cuenta de las múltiples negociaciones, alianzas y tensiones que trajo consigo estaconsistencia urbana.Algunas investigaciones hananalizado las consecuencias del desarrollo industrial y de la proletarización de la sociedad urbana, a través de los conceptos y categorías ofrecidas a hombres y mujeres bajo el capitalismo industrial.Por ejemplo, para inicios del siglo XX la asociación entre trabajo femenino y comercio sexual, como señala Elizabeth Hutchinson, creció a tal punto que ambas actividades se volvieroncasi indistinguibles (Hutchinson, 1998, 131).

Podemos plantear que para el contexto chileno el desarrollo urbano y fabril reforzó nuevasalianzas entre enfermedad mental e incapacidad, como resultado no solo de los marcos legales y médicos, sino de las exigencias y condicionesde la modernidad. El darwinismo social instaló la posibilidad del atavismo, de una regresión que posicionó al "degenerado» como opuesto del ciudadano. Así, el progreso, transparentó sus costos y problemáticas, junto a sus posibles regresiones. En esta tensión el ciudadano fue considerado una pieza del desarrollo, pero a su vez, un posible obstáculo, el cual debía ser medido, etiquetado, estudiado y categorizado, para situar y ordenar a quienes parecían no ajustarse a los requerimientos del ideal modernoimpulsado por el capitalismo industrial, entre ello los incapaces.

Las nuevas condiciones del hogar y del vecindario influyeron tempranamente en la caracteriza- ción de la enfermedad mental y en su vinculación con la incapacidad. Su densidadreformuló los significados de la desviación y las respuestas a su administración. Así, el hogar urbano enfrentó la enfermedad de nuevos modos. Recibió la oferta terapéutica y demandó el ingreso de los enfermos a instituciones asilares, como la Casa de Orates de Santiago (1852) o los diversos hospicios y hospitales que se levantaron en el país durante el siglo XIX. El desarrollo de la medicina y de sistemas curativos más eficaces llevaron a considerar a la locura no tanto como un destino, sino como una condición posiblede modificar. En este contexto la enfermedad y con ella la incapacidad cobraron nuevos significados, siendo entendidos como momentos en la vida de algunas personas, estados reversibles y cambiables.

Las familias y los vecinos explicaron las dificultades que provocaba el comportamiento enajenado de un enfermo, en un momento en que la vida parecía volverse cada vez más compleja. El encierro terapéutico fue recibido con alivio por quienes buscaban separar a los enfermos de los peligros de la vida moderna y también reducir el peso de su cuidado. Como explicaba la familia De la Fuente, en el juzgado de Valparaíso, el interés por internar a uno de sus miembros en la Casa de Locos, respondía a los obstáculos y peligros que ofrecía la ciudad hacia 1876. "Los ferrocarriles" que cruzaban el puerto, representaban un constante riesgopara el enfermo, lo que obligaba a que una persona lo acompañara y vigilara constantemente ${ }^{11}$. Pero también, la Casa de Orates representaba un descanso y una necesidad para aquellos, que asediadospor la locura, dejaban de confiar en los recursos cotidianos usados para su control y tratamiento.

Así la vida urbana mostraba un rostrohostila los enfermos, mientras las nuevas instituciones hospitalarias se presentaban como alternativas no solo para el control terapéutico, sino para la validación y cuidado de la demencia. La Casa de Locosofre-

AJV, Interdicción, 1876, L 697, p 21, f. 2. 
ció por medio del tratamiento moral la posibilidad de revertir la cualidad de demente e incapaz, y de solucionar algunas de las fatalidades de la enfermedad.Con el paso del tiempo, el desarrollo de nuevas tecnologías terapéuticas reforzó su destino e impulsó el desplazamiento de la familia como principal espacio de cuidado y redención del enfermo, al menos en el ámbito discursivo de la primera mitad del siglo XX.

Pese a la gradual penetración de la institución manicomial como agente de definición de la locura, su influjo fue menor para la segunda mitad del siglo XIX. La densidad urbana mostró la importancia de las redes familiares y vecinales en la identificación de la desviación. Los familiares tendieron a ser los primeros en acusar una demencia, sea con el objeto de solicitar la internación en el asilo de locos o de obtener la interdicción del enfermo. Padres, madres, hermanos, hijos y tíos, entre otros, se presentaron como voces autorizadas para denunciar la enfermedad mental y solicitar la oficialización de una incapacidad.En ese proceso, visibilizaron la carga política del hogar, mostrando comoel cumplimiento o incumplimiento de los roles y compromisos domésticos permitía distinguir al capaz del incapaz, al hábil del inhábil y al competente del incompetente.

Junto a los familiares, los vecinos acudieron a los juzgados con frecuencia para presentar testimonio del comportamiento de sus cercanos. El haber estado "observándolos constantemente", como decían muchos de ellos, resultado de la cercanía y del roce cotidiano, les permitía aportar con una versión autorizada de los hechos. Así, se hicieron parte de las imputaciones de locura, mostrando como la caracterización de la enajenaciónescapaba a la familia ytocaba a un grupo más amplio de la población.Participaron en los procesos judiciales por distintos motivos, pero principalmente porque la fricción y la cercanía urbana los hacía receptores de los abrazos y los golpes, yoídos de las risas y los gritos. Se topaban con desórdenes y transgresiones, y cumplían un papel substancial enla lectura de los comportamientos. Así, por ejemplo, las "palabras inmorales" pronunciadas poruna jo- ven madre y sus continuos "desórdenes"habían perturbado profundamentehacia 1881 a un barrio de Valparaíso, a tal punto que sus vecinosconcurrieron a tribunales a ratificarsu comportamiento, respaldar su interdicción y sugerir su encierro en la Casa de Orates. Los hechos como relataba otro cercano, se repetían constantemente hacía ya casi dos años y dada esta reiteración, la policía ya no hacía caso a sus llamados ${ }^{12}$. También participaban en las imputaciones infundadas, mostrando como la cercanía también les permitió rechazar demencias e incapacidades, defendiendo "la honradez", "juiciosidad", "laboriosidad" y "contracción para el trabajo" de aquellos vecinos "apreciados" y "honorables".

La vida moderna instaló nuevos estándares de capacidad en la familia y fuera de ella, lo que tensionó los roles y los compromisos domésticos. Estosestándares sembraron dudas sobre la sanidad de quienes no se amoldaban a las nuevas velocidades, formatos laborales, presiones, sistemas administrativos, y a las varias complejidades de la vida contemporánea.En este ámbito, los cambios económicos cumplieron un papel central en acercar la enfermedad mental a la incapacidad.

Durante la segunda mitad del XIX, una serie de procesos promovieronmovimientos migratorios $y$ apoyaron la proletarización del trabajo rural. También impulsaron la monetización de la sociedady la reducción del intercambio, modificando paulatinamente las formas de organización económica de las familias chilenas.Así, las discusiones en torno a la enfermedad se tiñeron de la problemática de la capacidad de ordenar el capital y el patrimonio, de administrar el salario, de hacerse cargo de una economía familiar monetizada. Este cambio supuso nuevos desafíos que, podemos plantear, impulsaron el uso de la interdicción, y de paso el reforzamiento entre locura e incapacidad.

Los expedientes de interdicción muestran que la enfermedad mental y el estatuto de incapacidad se relacionó estrechamente conprácticas patrimo-

AHNCh, AJV, Interdicción, 1881, L. 1168, p. 13, f. 3. 
niales y financieras juzgadas como impropias. En una gran mayoría de los casos, la locura fue comunicada y la incapacidad acusada cuando el imputado se mostraba incompetente de administrar su propio patrimonio o el de su familia. Los legistas explicaban que para determinar la capacidad civil de un imputado por demencia se debía evaluar si "tiene una correcta apreciación de las cosas y de sus consecuencias" y por sobre todo, "que la enfermedad mental no lo inhabilite para administrar completamente sus negocios" (Álvarez, 1901, 400).

Santiago y Valparaíso concentraron infraestructura financieray lideraron la mercantilización de la sociedad chilena, seguidos por reductos industriales más pequeños desplegados por el país. En estos espacios las familias acusaron tempranamente el descalabro económico que provocaba la demencia de uno de sus miembros, utilizando al mismo tiempo sus acciones y omisiones como prueba de su enfermedad.»Son actos de persona cuerda i prudente arrendar todas sus propiedades incluso la casa en que vive, i darse por recibida de arriendos que jamás se han percibido?» ${ }^{13}$, preguntaba a la justicia Manuel Valdés, hijo de Isidora Segura en Valparaíso hacia 1887. Corresponde firmar "una escritura publica, vendiendo seis barras de la mina "Carmen" por seis mil pesos, esto és la cuarta parte de su valor" inquiría Elisa Navarrete, respecto al comportamiento de su marido Julio Herrera en Iquique hacia $1891^{14}$.También atribuían el despilfarro y la prodigalidad como comportamientos inherentesa la locura. Como acusaba Elisa Navarrete respecto a su marido en Iquique hacia 1891, “¿Que há hecho de este dinero? Lo que hace todo loco o que tiene su juicio adormecido (...) ¿Lo habrá votado, regalado o dilapidado y mientras tanto yo tengo que mendigar fuera para dar al albergue y alimentar mis seis hi-

13 AJV, Hijopideinterdicción de sumadreporbienes en herencia del fallecido padre, 1887, L 1380, p 2, f. $2 \mathrm{v}$.

14 AJI, Interdicción, 1891, L 786, p. 1, f. 9v. jas, todas mujeres... ¡Que tal el juez! i será esto posible?» ${ }^{15}$.

Así la capitalización de la sociedad chilena generaba dos procesos paralelos. Por un lado impulsaba nuevos modos de caracterización de la enfermedad mental asociándola a la incorrecta administración del dinero, a la dilapidación y a falta de habilidad financiera. Por otro, mostraba la masificación de la práctica de interdicción como herramienta para administrar la enfermedad mental, lo que conllevaba a su vez a la homologación judicial del demente con el incapaz. Los bienes fueron elementos claves en la utilización de la interdicción para la administración de la locura y particularmente aquellos bienes monetizados que parecían ser elementos mucho más volátiles y susceptibles a sufrir los baches de la mente insana. Los demandantes acudieron a la justicia para intentar cuidar no solo al demente, sino sus depósitos bancarios, los montepíos, las herencias, los salarios y los arrendamientos. Resulta interesante además constatar que la necesidad de ordenar por medio de la interdicción el patrimonio familiar alborotado por comportamientos indeseados, no fue prerrogativa de la elite, como consecuencia de una sociedad que expandía su sistema económico y el cuerpo de trabajadores asalariados.

\section{Ideas al cierre: enfermedad, discapacidad y paso al capitalismo}

Estudios recientes han mostrado que "tener una deficiencia significa ser -potencialmenteportador de un atributo descalificador y un estigma" que posiciona a hombres y mujeres en una posición "devaluada que pone en cuestión su pleno carácter humano" (Ferrante, 2013, 100).La historia complementa esta mirada exponiendola historicidad de la marginación asociada a la diferencia y la necesidad de repensar los procesos,

15 All, Interdicción, 1891, L 786, p. 1, f. 9v. 
contextos y necesidades que han guiado su administración.

En los últimos años los llamados disabilitystudies handevelado el protagonismo histórico de la "discapacidad" y sus distintas expresiones; al mismo tiempoque han levantado preguntas y ofrecido análisis que han obligado a reconocer la importancia de la diferencia corporal y mental en el estudio del pasado. Como ha planteado Douglas Baynton, pese a que los historiadores se han volcado en las últimas décadas con voracidad al estudio de la construcción de la desigualdad, sea a través de categorías como la clase, el género o la etnia, pocos se han detenido en el estudio de los conceptos de marginación vinculados al concepto de disability (Bayton, 2001, 33).Así también, pese a la importancia de la capacidad en la definición de la ciudadanía, poco se ha dicho de esta relación, más aun desde lo patológico.

La "discapacidad" fue central en los tres grandes debates sobre ciudadanía - sufragio femenino, afroamericanos y políticas de inmigración. Como señaló Baynton, el cuestionamiento dela ciudadanía estuvo acompañado de la entrada de la discapacidad a escena, como medida para clarificar y definir quién podía gozar de la ciudadanía y quién merecía ser excluido de ella (Bayton, 2001, 33). Así, conceptos como "incapacidad" han servido para justificar la inequidad y para respaldar acciones de ordenamiento social que han arrastrado consecuencias simbólicas y materiales en la vida de hombres y mujeres.

La interdicción, como práctica social, nos permite adentrarnos en algunas de las lógicas de exclusión y ordenación implementadas en Chile durante la segunda mitad del siglo XIX a través del vinculación de la demencia con la incapacidad. Las historias de más de mil personas declaradas dementes e incapaces como resultado de la comprobación judicial de una interdicción ofrecen interesantes contextos de producción y significación de la marginación, así como de la disca- pacidad física, mental y emocional ${ }^{16}$. El estudio de cientos de expedientes judiciales de interdicción, da cuenta del peso de la enfermedad en un momento significativo en la historia chilena, caracterizado por la conformación de disciplinas médicas que intentaban mapear el efecto fisiológico de las patologías mentales. Esa organización disciplinaria, pese a rechazar algunos de los antiguos estados absolutos de insania y ofrecer la posibilidad de la recuperación por medio de una batería de procedimientos terapéuticos, subrayó la desposesión en la que se encontraba el sujeto enfermo, naturalizando la deficiencia y oficializando la acción etiquetadora de la agencia médica. Paralelo a estos procesos, el ordenamiento ciudadano propuesto por el Estado en los textos constitucionales y luego en los códigos legales, instaló la eventualidad de la marginación, producto de la "discapacidad", rotulando al sano como ciudadano capaz y al enfermo como incompetente.

Junto al saber médico y los cuerpos legales, los parientes y vecinos se constituyeron como los principales agentes en la imputación de demencia. Resulta interesante constatar que fueron ellos quienes nutrieron de sentido a la enfermedad mental, entregando relatos que materializaban la patología -la mayor de las veces invisible al ojo experto- por medio de su asociación con la incapacidad. En esta alianza, el contexto emergió como elemento clave para situar el espesor de la enfermedad y la dimensión del problema. La falta de capacidad resumió en un importante número de casos, la enfermedad. La imposibilidad de hablar, de oír, de moverse; la dificultad para mantener afectos, cuidar cariños, conservar la compostura, comportarse en público, enseñar las emociones; los obstáculos en la administración de los negocios, en el entendimiento de los códi-

16 Aludimos a 1000 casos identificados, suponemos que este número fue más alto si consideramos a quienes enfrentaron cuestionamientos a su capacidad, pero que no alcanzaron a recibir una declaración de interdicción. 
gos económicos, en el conocimiento de los formatos bancarios, fueron sindicados como códigos de incapacidad y como signos de demencia.Entre estos, aquellos problemas relacionados con la administración del capital y la reproducción social de la familia, cobraron mayor visibilidad y urgencia, acelerando la judicialización de la demencia y mostrando la correspondencia entre la falta de adecuación al modelo económico y la incapacidad. Estas constataciones dejan abiertas preguntas sobre la actual vinculación entre la mercantilización, la enfermedad y la incapacidad, así también entre los objetivos económicos y la marginación de ciertos grupos producto de la patologización de sus comportamientos.

Durante los últimos doscientos años se gestó un doble proceso que reubicó tanto al saber médico, como a sus profesionales y diagnósticos, en su relación con el Estado y con la sociedad. En relación a la locura, las distintas teorías que explicaban las enfermedades mentales reforzaron el vínculo de algunas de estas condiciones con la noción de incapacidad -luego discapacidad-. También, la proliferación de patologías y la "psicologiación" y "psiquiatrización" de la sociedad, Ilevaron a considerar a las afecciones mentales como elementos inherentes a la vida moderna.

Estos procesos dejan ver una paradoja aun presente. Por un lado la medicina moderna y las disciplinas que se abocaron al estudio de lo mental, enseñaron que la locura tomaba variadas formas, que no acechaba siempre al intelecto y que podía remitir con una adecuada terapéutica. Sin embargo, pee a este discurso que masificó la experiencia de locura e insistió que todos podían tener la potencialidad de enfermar, las ambiciones y complejidades de la vida moderna reforzaron la vinculación de la enfermedad mental con la incapacidad.

Estos procesos dejan ver también que conceptos tales como "incapacidad" o "discapacidad" corresponden a productos históricos, que se desplazan, modifican y se disimulan, producto de contextos que los reconocen y posicionan o desplazan y silencian. Los cuerpos legales de la temprana República establecieron las fronteras del quehacer individual al definir una estructura diferenciada para aquellas personas identificadas como incapaces y otorgaron significados legales y sociales a dichas categorías. Estas regulaciones entregaron una primera definición de la capacidad individual y posicionaron, a través de su usojudicial, a sujetos comunes como constructores de categorías y explicaciones más precisas sobre incompetencia vinculadas a nociones médicas de patología mental. Reconociendo estos usos, resulta necesario reconocer su historicidad para comprender los procesos de formación e instalación de estas categorías, y para pensar como hoy les damos sentido y destino.

\section{REFERENCIAS BIBLIOGRÁFICAS}

Alvarez, A., "La Incapacidad Mental ante la Medicina Legal y ante los principios de la Legislación Comparada", Primer Congreso Médico Latino-Americano, Tomo I, Santiago, Imprenta, Litografia, Ecuadernacion Barcelona, 1901; 399-421.

AnuarioEstadístico de la República de Chile correspondiente al año 1867, Santiago, ImprentaNacional, 1868.

Baynton, D., "Disability and the Justification of Inequality in American History", en Paul Longmore y LauriUmansky (eds.), The New Disability History: American Perspectives, New York, New York University Press, 2001.

Bello, A., Los 50 artículos del Código Civil explicadosporsuautor, Valparaíso, Imprenta del Mercurio, 1874.

Blest, G., Ensayosobrelascausas mas comunes y activas de lasenfermedadesque se padecen en Santiago de Chile con indicaciones de los mejoresmediosparaevitarsudestructorainfluencia, Santiago, Imprenta de R. Rengifo, 1828.

Brito, A., "Del rancho al conventillo. Transformaciones de la identidad popular femenina, Santiago de Chile, 1850-1920", Godoy, L., (et. al) Disciplina y desacato.Construcción de identidad en Chile, siglos XIX y XX, Santiago, SUR/Cedem, 1995.

Bruner, J.J., "Fragmentos de unahigienepública de Santiago", Anales de la Universidad de Chile, 1857

Cansanello, C., De Súbditos a Ciudadanos. EnsayoSobrelasLibertades en los OrígenesRepublicanos. Buenos Aires 1810-1852, Buenos Aires, Imago Mundi, 2003.

Collier, S., Chile: The Making of a Republic, 1830-1865, Cambridge, Cambridge University Press, 2003.

Constitución de la República de Chile, Santiago, Imprenta de la Unión, 1833.

Correa, M.J., "Lo lego en la negociación de la autoridad médico judicial en Santiago de Chile, 1890-1892. Trinidad Álvarez y su idiotismo congénito", Sudhistoria, No 5, Julio-Diciembre, 2012. 
Correa, M.J., Historias de Locura e Incapacidad. Santiago y Valparaiso (1857-1900), Santiago, ActoEditores, 2013.

Correa, M.J.,Lay People, Medical Experts and Mental Disorders. TheMedicalization of Insanitythroughthelncapacitation of theMentaIlylll, 1830-1925. Tesis doctoral no publicada, doctor of Philosophy, UniversityCollege London, 2012.

De la Plata, E., Observacionessobre la InterdicciónporDemencia, Santiago, Imprenta de la Unión, 1887.

De Ramón, A., "La población informal. Poblamiento de la periferia de Santiago de Chile, 1920-1970", EURE 1990, 50; 5-17.

De Ramón, A., Santiago de Chile, Sudamericana, Santiago, 2000.

Díaz, W., Medicina.De la manera de aprenderienseñar la Medicina, en susrelaciones con el progreso de la cienciai con lasventajasquepuedenreportar a nuestra patria, Anales de la Universidad de Chile, 1863; 437-457.

Elguero, J. R., "Informe del Médico de la Casa de Locos", Memoriaque el Ministro de Estado en el departamento del Interior presenta al CongresoNacional de 1863, Santiago, ImprentaNacional, 1863.

Ferrante, C. y Ferreira, M., "Cuerpo, discapacidad y trayectoriassociales: dos estudios de casocomparados", Revista de Antropología Experimental, 2008, 8; 403-428.
Ferrante, C., "Cuerpo, discapacidad y menosprecio social. Ajustes y resistencias a lastiranías de la perfección", en Aguila, L.M. (et. al. ed.), Discapacidad, Justicia y Estado.Discriminación, estereotipos y toma de conciencia (Buenos Aires, Infojus, 2013); 99-122.

Hutchison, E., "'El frutoenvenenado del árbolcapitalista': Women Workers and the Prostitution of Labor in Urban Chile, 1896-1925", Journal of Women's History, 1998, Vol. 9, núm. 4; 131-151.

Memoria que el Ministro de Estado en el departamento del Interior presenta al Congreso Nacional de 1859, Santiago, Imprenta Nacional, 1859.

Molina, E. "El concepto de ciudadanía en el discurso y en lasprácticas de las "modernas" formas de sociabilidadasociativa (18101813)", Sociohistórica, 2002, 11-12; 135-157.

Rengifo, F., "Familia y escuela. Unahistoria social del proceso de escolarizaciónnacional.Chile, 1860-1930", Historia, 2012, 45, vol.I; $123-170$.

Sábato, H. (ed.), CiudadaníaPolítica y Formación de lasNaciones. PerspectivasHistóricas de América Latina, Buenos Aires, Fondo de CulturaEconómica, 1999.

Stiker, H., A History of Disability, Michigan, The University of Michigan Press, 1999.

Valdes, E., "La Asistencia Publica en Chile", Anales de la Universidad de Chile, 1892-1893; 1139-1171. 